\title{
Antibiotic therapy-induced collateral damage: IgA takes center stage in pulmonary host defense
}

\author{
Juergen Lohmeyer, ${ }^{1}$ Rory E. Morty, ${ }^{1,2}$ and Susanne Herold ${ }^{1,3}$ \\ 'Department of Internal Medicine II, University of Giessen and Marburg Lung Center (UGMLC), member of the German Center for Lung Research (DZL), Justus-Liebig-University, Giessen, Germany. \\ 2Department of Lung Development and Remodelling, Max Planck Institute for Heart and Lung Research, member of the German Center for Lung Research (DZL), Bad Nauheim, Germany. \\ ${ }^{3}$ Division of Pulmonary and Critical Care, Northwestern University Feinberg School of Medicine, Chicago, Illinois, USA.
}

The use of broad-spectrum antibiotics in empirical antimicrobial therapy is a lifesaving strategy for patients in intensive care. At the same time, antibiotics dramatically increase the risk for nosocomial infections, such as hospital-acquired pneumonia caused by Pseudomonas aeruginosa, and other antibiotic-resistant bacteria. In this issue of the $J C l$, Robak and colleagues identified a mechanism by which depletion of resident gut and lung microbiota by antibiotic treatment results in secondary IgA deficiency and impaired anti-P. aeruginosa host defense. Impaired defenses could be improved by substitution of polyclonal IgA via the intranasal route in a mouse model of pneumonia. Importantly, antibiotic treatment caused lung IgA deficiency that involved reduced TLR-dependent production of a proliferation-inducing ligand (APRIL) and B cell-activating factor (BAFF) in intensive care unit patients. These patients might therefore benefit from future strategies to increase pulmonary IgA levels.

\section{Antibiotic use and risk of nosocomial infection}

Broad-spectrum antibiotics $(\mathrm{ABx})$ form the backbone of empiric antimicrobial treatment regimens, which are lifesaving for those with severe infections such as pneumonia, peritonitis, or sepsis, and which constitute an integral part of treatment guidelines (1). However, apart from driving the selection of multiresistant pathogens, $\mathrm{ABx}$ severely disrupt the host microbiome, which has been increasingly recognized as a critical factor in shaping host immunity (2). Antibiotic stewardship and improved point-of-care microbial diagnostics increasingly facilitate the optimized use of specific antibiotics (3), but in critically ill patients the empiric use of $\mathrm{ABx}$ will remain indispensable. Point-prevalence studies have documented that to date $64 \%-71 \%$ of all patients in intensive care units (ICUs) receive antibiotics $(4,5)$. Previous antimicrobial therapy is a major risk factor for the normal microbiome to turn into a so-called pathobiome, which is diagnosed as hospital-acquired pneumonia (HAP) and often associated with outgrowth of $P$. aeruginos $a$ or other antibioticresistant bacteria $(6,7)$. Recent estimates indicate that in Europe, 2.6 million cases of hospital-acquired infections occur each year, and that HAP accounts for the highest disease burden and number of deaths (8). HAP dramatically increases both the length of the hospital stay and health care costs, and is associated with a mortality of up to $13 \%$ (9).

The increased susceptibility of critically ill patients to infection with multidrug resistant (MDR) gram-negative bacteria was originally attributed to generation of a permissive niche with selection of MDR pathogens that were not covered by the $\mathrm{ABx}$ regimen $(10,11)$. In this issue, Robak and colleagues (12) identify an additional mechanism by which lung and gut micro-

Related Article: p. 3535

Conflict of interest: The authors have declared that no conflict of interest exists.

Reference information: / Clin Invest. 2018:128(8):3234-3236. https://doi.org/10.1172/JCI122032.

biota changes induced by $\mathrm{ABx}$ treatment inhibit immunoglobulin A (IgA) production in the lung, thereby increasing susceptibility of ABx-treated patients to infection with $P$. aeruginosa (Figure 1 ).

\section{Microbiota-host immunity cross-talk}

How do the findings of Robak et al. help expand our understanding of the interplay between the microbiota and host defense in lung infection, and thereby promote development of new prophylactic or therapeutic strategies? It is well established that antimicrobial therapies inevitably cause dramatic and long-lasting collateral damage to the diverse populations of commensal bacterial species that are part of a patient's intestinal microbiota. However, recent investigations have further revealed that composition of the microbiota also crucially impacts systemic and pulmonary innate immune responses during bacterial and viral infections (13-15). Considerably less is known about how the microbiota ecosystem and its depletion by $\mathrm{ABx}$ impact adaptive immune responses. The gut microbiota is critical for regulating intestinal IgA production, as IgA-secreting cells and IgA production are almost absent in the gut of germ-free mice (16). Moreover, microbial signals are known to activate TLRs on intestinal epithelial cells and DCs to induce production of the crucial $\mathrm{B}$ cell survival signals APRIL and BAFF, which in turn, promote IgA production by plasma cells (17-19). In addition, microbiotaderived short-chain fatty acids have recently been demonstrated to positively regulate IgA production (20).

\section{IgA-mediated pulmonary} host defense

What makes IgA so special for lung host defense? Immunoglobulin A has a critical role in immune defense, particularly at mucosal surfaces, and IgA is specially 


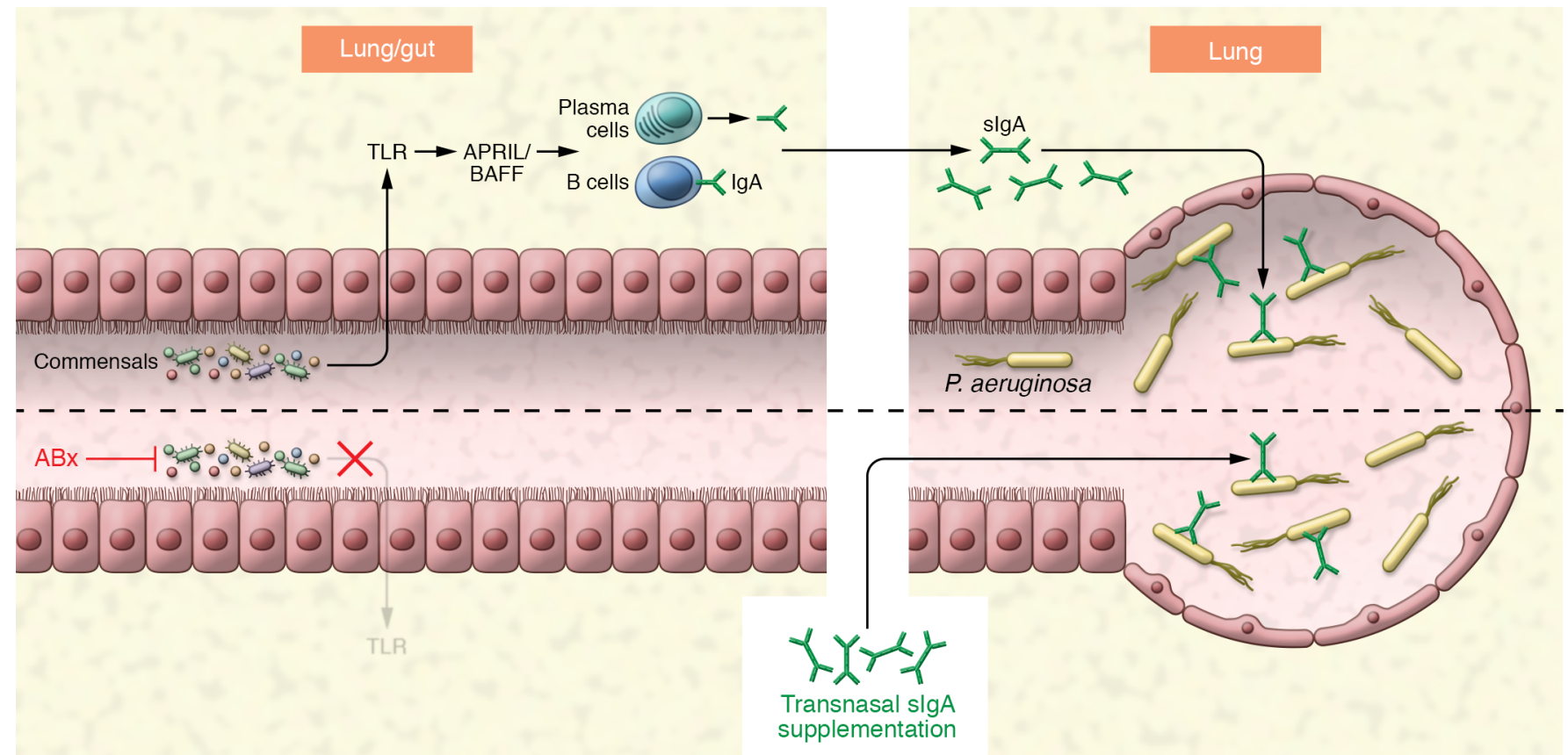

Figure 1. Broad-spectrum antibiotic treatment exerts severe collateral damage by inhibiting microbiota-induced secretory IgA synthesis and IgAdependent lung host defense toward $\boldsymbol{P}$. aeruginosa. The lung and gut commensal microbiota induce lgA production at mucosal surfaces involving TLR-, APRIL-, and BAFF-dependent signaling. Lung secretory IgA (slgA) binds $P$. aeruginosa and reduces host susceptibility to $P$. aeruginosa pneumonia. ABx treatment destroys luminal microbiota and severely reduces slgA production by lung IgA-secreting plasma cells, thereby impairing anti-pseudomonas host defense, which can be reestablished by transnasal administration of slgA.

equipped to undertake this task through the unique structural attributes of the IgA heavy chain and by virtue of its ability to polymerize (16). Notably, more IgA antibodies are synthesized in mammals per day than antibodies of all other isotypes combined. IgA plays a pivotal role in maintaining a homeostatic relationship between the host and the resident microbiota of the intestine (21). Production of high-affinity and antigen-specific IgA in Peyer's patches and mesenteric lymph nodes has been documented to be T cell dependent (16), whereas low-affinity polyreactive IgA responses are mainly induced in isolated lymphoid follicles and in subepithelial B cells in a T cellindependent manner (19). Both low- and high-affinity IgA regulate the composition of the intestinal microbiota by coating many of the bacterial components in the intestinal lumen, in order to maintain intestinal homeostasis (21).

In contrast to the gut, it was not known whether microbiota-dependent IgA production also maintained lung microbiome homeostasis and whether IgA was required for antibacterial defense in the lungs of mice and humans. Moreover, it had not been investigated if and how disturbances to this mechanism contribute to the enhanced susceptibility to lung infections following antibiotic treatment. The findings by Robak et al. explain how depletion of the resident microbiota affects lung host defense against $P$. aeruginosa and represent a fundamental step in filling this knowledge gap. To our knowledge, this is the first study to demonstrate that depletion of resident microbiota by $\mathrm{ABx}$ inhibits TLR-dependent production of APRIL, resulting in secondary IgA deficiency in the lungs of both mice and ICU patients and increases susceptibility to subsequent $P$. aeruginosa infection (Figure 1). There are several essential unresolved questions that arise from this work. First, what is the cellular source of TLR-dependent APRIL, $\mathrm{BAFF}$, and IgA production? The relevant cell might in fact be in the lung, as suggested by a recent study that demonstrated that lung $\mathrm{CD}_{103}{ }^{+}$and $\mathrm{CD} 24^{+} \mathrm{CD} 11 \mathrm{~b}^{+} \mathrm{DCs}$ induce IgA class-switch recombination by activating $\mathrm{B}$ cells through $\mathrm{T}$ cell-dependent or -independent pathways (22). This process involved microbial stimuli acting through MyD88/TRIF-mediated TLR signaling and TGF- $\beta$ receptor signaling.
However, it is also conceivable that the induction of IgA generation occurs in the intestinal tract and that $\operatorname{Ig} \mathrm{A}^{+}$plasma cells subsequently migrate to the lung, given that migration of plasma cells between lung and intestine has been previously described (22). Second, the cellular compartment responsible for the TLR-dependent APRIL production and the specific TLR member involved remain unknown. Third, the mechanism by which IgA exerts antimicrobial properties directed against $P$. aeruginosa (and most likely other bacterial species) in the lung is unknown, but likely is similar to the gut, where IgA achieves neutralization of invasive pathogens by various mechanisms, such as immobilization, opsonization, or killing $(23,24)$. Knowledge about such molecular mechanisms will be crucial to select the most appropriate preparations for passive IgA supplementation. Finally, the specific features of respiratory and/or intestinal microbiota (for example, distinct bacterial taxa) required for the induction of protective immunity in the lung, and the potential of probiotics to rescue this IgAdependent antibacterial defense, remain to be defined. 


\section{Conclusions and future directions}

Current attempts to target the affected microbiome for infection prevention or even treatment primarily intervene at points upstream to reconstitute the microbial flora or/and microbial-derived signals using live microbiota (probiotics) or even fecal transplantation; however, these strategies face considerable regulatory hurdles (11). The pathway outlined by Robak et al. (12) now offers an attractive downstream intervention to compensate for disrupted microbiome-related defects in humoral defense. To optimize a passive IgA supplementation approach toward clinical translation, the potential of different immunoglobulin preparations and routes of administration as preemptive or therapeutic interventions for HAP need to be evaluated in both experimental models and clinical trials. Although the location of TLR-dependent microbiota sensing and APRIL, BAFF, and IgA induction remains to be defined, the pathway identified by Robak et al. also holds promise for the development of novel strategies that rely on preserving or restoring $\operatorname{IgA}$ function under $\mathrm{ABx}$ treatment. IgA restoration could be achieved by engagement of potentially druggable candidate pathways using suitable agonists of the still-undefined TLR signaling pathway or the APRIL/BAFF signaling axis. Such treatments are not only envisioned to improve outcomes of $P$. aeruginosa-induced $\mathrm{HAP}$, but might also be applied to reduce chronic $P$. aeruginosa colonization in those at risk, such as patients with cystic fibrosis.

\section{Acknowledgments}

This work was supported by the Deutsche Forschungsgemeinschaft (DFG) through grants EXC147 and CRU309 (P2, P8, P6), the German Center for Lung Research (DZL), and by the Max Planck Society.

Address correspondence to: Juergen Lohmeyer, Department of Medicine II, JustusLiebig-University, Klinikstr. 33, 35392 Giessen, Germany. Phone 49.641.985.57061; Email: juergen.lohmeyer@innere.med. uni-giessen.de.

1. Ferrer R, et al. Empiric antibiotic treatment reduces mortality in severe sepsis and septic shock from the first hour: results from a guideline-based performance improvement program. Crit Care Med. 2014;42(8):1749-1755.

2. Honda K, Littman DR. The microbiota in adaptive immune homeostasis and disease. Nature. 2016;535(7610):75-84.

3. Campion M, Scully G. Antibiotic use in the intensive care unit: optimization and de-escalation [published online ahead of print January 1, 2018]. J Intensive Care Med. https://doi. org/10.1177/0885066618762747.

4. Zarb P, et al. The European Centre for Disease Prevention and Control (ECDC) pilot point prevalence survey of healthcare-associated infections and antimicrobial use. Euro Surveill. 2012;17(46):20316.

5. Vincent JL, et al. International study of the prevalence and outcomes of infection in intensive care units. JAMA. 2009;302(21):2323-2329.

6. Shindo Y, et al. Risk factors for drug-resistant pathogens in community-acquired and healthcare-associated pneumonia. Am J Respir Crit Care Med. 2013;188(8):985-995.

7. Venier AG, et al. Identifying new risk factors for Pseudomonas aeruginosa pneumonia in intensive care units: experience of the French national surveillance, REA-RAISIN. J Hosp Infect. 2011;79(1):44-48.

8. Cassini A, et al. Burden of six healthcareassociated infections on European population health: estimating incidence-based disabilityadjusted life years through a population prevalence-based modelling study. PLoS Med. 2016;13(10):e1002150.

9. Melsen WG, et al. Attributable mortality of ventilator-associated pneumonia: a metaanalysis of individual patient data from randomised prevention studies. Lancet Infect Dis. 2013;13(8):665-671.

10. Buffie CG, Pamer EG. Microbiota-mediated colonization resistance against intestinal pathogens. Nat Rev Immunol. 2013;13(11):790-801.

11. Pamer EG. Resurrecting the intestinal microbiota to combat antibiotic-resistant pathogens. Science. 2016;352(6285):535-538.

12. Robak OH, et al. Antibiotic treatment-induced secondary IgA deficiency enhances susceptibility to Pseudomonas aeruginosa pneumonia. JClin Invest. 2018;128(8):3535-3545.

13. Clarke TB, Davis KM, Lysenko ES, Zhou AY, Yu Y, Weiser JN. Recognition of peptidoglycan from the microbiota by Nod1 enhances systemic innate immunity. Nat Med. 2010;16(2):228-231.

14. Ichinohe $\mathrm{T}$, et al. Microbiota regulates immune defense against respiratory tract influenza A virus infection. Proc Natl Acad Sci US A. 2011;108(13):5354-5359.

15. Abt MC, et al. Commensal bacteria calibrate the activation threshold of innate antiviral immunity. Immunity. 2012;37(1):158-170.

16. Pabst O. New concepts in the generation and functions of IgA. Nat Rev Immunol. 2012;12(12):821-832.

17. Tezuka H, et al. Regulation of IgA production by naturally occurring TNF/iNOS-producing dendritic cells. Nature. 2007;448(7156):929-933.

18. Litinskiy MB, et al. DCs induce CD40independent immunoglobulin class switching through BLyS and APRIL. Nat Immunol. 2002;3(9):822-829.

19. He B, et al. Intestinal bacteria trigger $\mathrm{T}$ cellindependent immunoglobulin A(2) class switching by inducing epithelial-cell secretion of the cytokine APRIL. Immunity. 2007;26(6):812-826.

20. Kim M, Qie Y, Park J, Kim CH. Gut microbial metabolites fuel host antibody responses. Cell Host Microbe. 2016;20(2):202-214.

21. Macpherson AJ, Yilmaz B, Limenitakis JP, Ganal-Vonarburg SC. IgA function in relation to the intestinal microbiota. Annu Rev Immunol. 2018;36:359-381.

22. Ruane D, et al. Microbiota regulate the ability of lung dendritic cells to induce IgA classswitch recombination and generate protective gastrointestinal immune responses. J Exp Med. 2016;213(1):53-73.

23. Simmons CP, et al. Central role for B lymphocytes and $\mathrm{CD}^{+} \mathrm{T}$ cells in immunity to infection by the attaching and effacing pathogen Citrobacter rodentium. Infect Immun. 2003;71(9):5077-5086.

24. Rollenske T, et al. Cross-specificity of protective human antibodies against Klebsiella pneumoniae LPS O-antigen. Nat Immunol. 2018;19(6):617-624. 